\title{
Theories for Use: On the Bearing of Basic Science on Practical Problems Martin Carrier (Bielefeld University)
}

Funding policies for science are usually directed at supporting technological innovations. The impact and success of such policies depend crucially on how science and technology are connected to each other. I propose an "interactive view" of the relationship between basic science and technology development which comprises the following four claims: First, technological change derives from science but only in part. The local models used in accounting for technologically relevant phenomena contain theoretical and non-theoretical elements alike. Second, existing technologies and rules of experience constitute another major repository of technological inventions. Third, technology dynamics is only weakly coupled to progress in basic science but it is closely related to science. There is a dependence of technological change on a more fundamental understanding, to be sure, but it is of an indirect and long-term character. Fourth, progress in basic research is sometimes the effect (rather than the cause) of technological change. Technological change sometimes brings about increased theoretical understanding (application innovation).

\section{Science Policy and the Advancement of Technology}

In the past half-century, scientific research has enjoyed financial support to an unprecedented degree. The reason behind this expenditure is not the hope for clarification of the nature of dark energy, nor the desire to learn more about supersymmetry. Underlying public and private sponsoring of research alike is the idea that science is a primary source of for technology development which is in turn viewed as a driving force of economic growth. In what follows I will consider science policies and funding strategies from the angle of such expectations. In other words, what could a science-policy be like that strengthens the technological productivity of science (leaving aside the ambivalent relationship between technological change and the labor market)? I attempt to highlight those features of scientific research that are relevant for technological innovation.

Projects of this sort thrive or fail on the appropriateness of distinguishing between kinds of scientific research. The distinction between basic or epistemic research, on the one hand, and applied research, on the other, is of foremost importance in this respect. It is objected occasionally that such a distinction cannot be sustained in that applied research, like basic research, produces new knowledge, and in that basic research, like applied research, has an impact on technology. The observation behind the objection is justified, to be sure, but merely of a limited bearing. It is still possible to conceptually separate basic and applied research by appeal to the goals pursued or, correspondingly, by the success criteria invoked. Epistemic research primarily strives for understanding natural phenomena or processes, applied research aims at practical needs or utility (Stokes 1997, 6-8). Correspondingly, the success of a project in applied research is assessed by economic standards whereas epistemic projects are judged according to the understanding gained. Such standard need not be assumed hypothetically, they are laid open publicly. Attempts to build 
optical switches or blue light emitting diodes (LEDs), to arbitrarily name a few technological challenges on the present agenda, are supported by estimates of the future potential market volume. Endeavors like the quest for the Higgs boson, by contrast, are justified by appeal to the human desire to understand nature's workings. The conceptual boundary between epistemic and applied research is marked by the commitment to understanding and utility, respectively. However, this distinction does not imply an empirical dichotomy; it does not rule out that a given research project serves both ends simultaneously.

Within the framework of this distinction between the basic and the applied, the question to be pursued here takes the following form: What is the role of theoretical knowledge or epistemic research in accomplishing technological innovations? In particular, can it be recommended that science policy sponsor thorough and broad theoretical analyses or are technological challenges be mastered better by concentrating on research that is directly connected to the particulars of the case at hand? To put it differently, is utility an outgrowth of understanding?

\section{The Cascade Model v. Emergentism}

The Scientific Revolution was fueled by the prospect of technological progress. Research into the laws of nature was claimed to be the royal road toward the betterment of the human condition. Conversely, seeking utility without understanding was considered a vain attempt. The power of intervention in the course of nature only accrues from disentangling the underlying causal fabric. Francis Bacon is the chief advocate of the principle that systematic basic research or epistemic penetration is the prerequisite of technology development. According to this cascade model (or "linear model") research striving for understanding is the chief source of technological change. The idea is that scientific knowledge flows smoothly from the highbrow principles to the practical solutions and becomes manifest, eventually, in useful devices. Practical tasks are best solved by bringing to bear insights into the mechanisms at work and understanding of the fundamentals (Bacon 1620, Bk. I., §3, §110, §117, §129; see Carrier 2006, 16, Carrier 2007, 22).

This traditional position was laid down and renewed in a highly influential way in a report Vannevar Bush delivered in 1945. Bush suggested basic research as the pivot of practical progress. As he argued, technological innovations are not likely to be generated by research narrowly targeted at the problem at hand. A more promising strategy is to conduct fundamental research whose technological fertility can be assumed to be superior for two reasons. First, the solution to a practical problem can arise as an unexpected consequence of a seemingly remote scientific principle. Second, practical progress is often reached through a novel combination of knowledge pieces. The two arguments entail that the theoretical resources apt for clearing up a practical difficulty cannot be established beforehand. Rather, practical success may be made possible by findings that are 
prima facie unrelated to the problem at hand. Conducting broad epistemic research creates the knowledge capital from which interests in the form of technological accomplishments are drawn (Bush 1945, Chap. 3).

After the Second World War this attitude was strongly dominant and formed the basis of research policies adopted by state institutions and industrial corporations. It was universally held that the advancement of a deeper scientific understanding was indispensible for a technological breakthrough and that the best strategy for stimulating the latter was promoting the former. As a result, industry-sponsored laboratories were set up which were devoted to basic research.

However, the intertwinement between basic research and technology development turned out not to be as close as anticipated. Historically speaking, science began to gain significance for engineering purposes not until the Second Industrial Revolution in the latter part of the $19^{\text {th }}$ century, and only in the $20^{\text {th }}$ century did the impact of science on technological change extend to larger parts of industry. Further, it happens that new technologies do not derive from scientific principles but rather build on existing technology or are due to tinkering at the bench. For instance, pharmacological research was for a long time (and in part still is) dominated by a methodology of trial and error. For instance, the most widespread procedure used in drug research throughout the $20^{\text {th }}$ century is schematic screening. A large number of potentially effective substances is administered to model organisms or tissue test systems and their effects are registered. When a successful medication had been tracked down by a procedure of this sort, it was in no way automatically clear how the drug operated. As a matter of fact, this is true of a significant fraction of the drugs in use today. Aspirin had successfully relieved headache for almost a century before its biological mechanism was finally disclosed. In such cases, medical progress did obviously not rely on understanding.

The progressive realization that technological change sometimes proceeds independent of progress in basic science generated a major change of attitude in the latter third of the $20^{\text {th }}$ century. It was no longer assumed that sponsoring pure research would guarantee cutting-edge technology. Among the early stages of this reorientation was a survey of the U.S. Department of Defense conducted in the late 1960s which found almost no connection between the physical operation of weapons systems then in use and more recent scientific discoveries (Stokes 1997, 55). As a result, funding policies generally switched to sponsoring targeted, short-term research projects which directly address specific problems.

Examples of this sort militate against the cascade model. In addition, developments in philosophy of science cast doubt upon the cascade model as well. Nancy Cartwright has drawn attention to the failure of universal laws to account for concrete phenomena with their rich details and 
variegated traits. Such laws and the highbrow theories they form part of are too abstract to capture the more subtle features of nature. They overgeneralize and thus lose touch with the richness of detail the phenomena exhibit. If the concrete experiences are supposed to be accounted for, generalizations of non-theoretical origin need to be part of the models (in addition to initial and boundary conditions). Rules of experience and specific assumptions bear the explanatory burden; tailormade approaches are needed when the experiences are to be addressed in their full complexity. Descriptive adequacy is limited to small-scale accounts; comprehensive theories inevitably lose touch with the phenomena. The patchwork quilt symbolizes the structure of scientific knowledge (Cartwright 1994, 322-323).

Such an approach may be termed "emergentist." Emergentists feature the specific character of the phenomena at each level of organization and deny that insights about the constituents will have much impact on the clarification of the properties of organized wholes. The emergentist position does not refer to applied science in the first place but rather to "applying science". The chief claim is that it is highly non-trivial to hook up theory with evidence and that the only way to get a grip on the phenomena is by making use of specific models that are tightly locked onto a particular problem. Still, emergentist approaches are tied up with a particular conception of applied research. The cascade model is abandoned; basic research is said to be largely unsuccessful in meeting applied challenges. Practical challenges should be addressed by doing research on precisely these practical challenges rather than directing research to the elucidation of the underlying principles and mechanisms (Carrier 2004b, 1-2; Carrier 2006, 18-19; Adam, Carrier \& Wilholt 2006, 438).

Thus we are faced with two contrary views on the relationship between basic and applied research. The general contrast between the cascade and the patchwork quilt brings a markedly different assessment of the bearing of fundamental theories on the solution of practical problems in its train. Consequently, the recommendations as to how applied research should proceed and which type of research should be supported are strongly at variance with each other.

\section{Local Models in Applied Research}

It has frequently been observed that — in contrast to the emergentist position — overarching theories do contribute essentially to explaining concrete phenomena. Yet such theories cannot bear the explanatory burden completely on their own; rather, models are needed as "mediators" between theory and evidence (Morrison \& Morgan 1999). The salient point is that the models typically used for applying theories are more complex than it was traditionally assumed in the philosophy of science. Such mediating models often do not only contain laws and boundary conditions but additional conceptual elements, such as generalizations from divergent theoretical sources or even without theoretical backing (that is, rules of experience), approximations and correction fac- 
tors, or parameters that can only be evaluated empirically (that is, read off from the data). Consequently, such models cannot be derived from highbrow theory. They rather rely to a considerable extent on extra-theoretical assumptions and their construction may involve a highly creative process. The "articulation" of a theory, the procedure of bringing to bear theoretical principles on concrete evidence, does in no way resemble a deductive chain but needs to resort to additional empirical, conceptual, and mathematical resources (Morrison 1999; Winsberg 2003; see Carrier 2004b, 9-13).

If the amount of adjustment of a model to the particular case in question is comparatively large, I speak of a "local model." Just to convey a rough idea, a model of the planetary system within Newtonian celestial mechanics is not local, a model of the spreading of Victoria perch within evolutionary theory is local. Much more empirical information, as inferred from the phenomena, is contained in the evolutionary model than in the mechanical one. Models are typically adjusted to a particular problem situation by parameter evaluations and correction factors, and a model is local if there are lots of specifics to be added and if they are restricted to a narrow scope. My claim is that applied science typically proceeds by constructing local models. The reason is that the variegated details of the phenomena typically escape the grip of comprehensive theory which merely addresses the generic features of the situation. Yet technology development is bound to take the quantitative particulars into consideration. Descriptive adequacy is often only accomplished by small-scale accounts as provided by local models.

However, the conceptual structure of the local models developed in applied research is typically still shaped by general theory. Applied research does in no way proceed on the exclusive basis of observational generalizations, experience-based regularities, ad-hoc assumptions, or rules of thumb. The conceptual backbone of such models, as a rule, derives from theory; the necessary adjustments are made by way of modifying this theory-based structure. Let me buttress this claim by giving an example.

"Giant magnetoresistance" is a physical effect discovered in 1988 and quickly explored by industrial research laboratories. The relevant arrays involve a sandwich-like structure in which two ferromagnetic semiconducting layers cover a non-ferromagnetic conductor layer in between. It was found that the electric resistance of such arrays is liable to large ("giant") variations, dependent on the orientation of the magnetization directions of the two ferromagnetic layers relative to each other. This magnetization direction can be influenced by an outside magnetic field so that the electric resistance of the array is affected by such a field. Consequently, its electric resistance indicates the intensity of a surrounding magnetic field. The effect is suitable for building extremely 
sensitive magnetic field sensors. Giant magnetoresistance underlies the functioning of today's magnetic read heads; it is used for hard disks or magnetic tapes.

The qualitative explanation of the effect was suggested immediately after its discovery. Quantum theory entails for a layered array of this sort that the scattering of electrons should depend on the orientation of the electron spin relative to the prevailing magnetic field. Electron scattering is the mechanism underlying electric resistance so that a relationship between resistance and field orientation ensues. Consequently, the basis of giant magnetoresistance is the theoretically well-understood spin-dependent scattering of electrons. However, this qualitative understanding did not also furnish automatically the pertinent quantitative relations. Knowledge of the precise dependence of the pertinent quantities is necessary for any practical use of the effect. If a read head is supposed to be constructed, the influence of layer thickness, material properties, temperature variations and changes of the magnetic field need to be known exactly. The anticipation of subtle dependencies of this sort transcended the scope of the theoretical account (Wilholt 2006, 72 79).

In order to get access to the details of the phenomenon, a local model needs to be built. Its conceptual structure reflects the theoretical account and yields consequences to the effect that the sensitivity of resistance changes essentially hinges on two parameters, namely, material properties and layer geometry (i.e., the thickness of the layer and the spatial dimensions of the system). The model also entails generic consequences as to the impact of certain such properties and arrays but leaves large room for empirical adjustment. In order to arrive at "design rules" for particular devices, a huge number of parameters need to be evaluated empirically and their impact on the quantities in questions be measured. When it came to figuring out the precise relations, as requisite for the construction of reliable contrivances, recourse to empirical adjustment was indispensible (Wilholt 2006, 79-80).

\section{Why Resort to Theories in Applied Research?}

Yet in spite of the limited grip of theory on the phenomena, theoretical understanding is useful in applied research. It is helpful to bring to bear theory on practical problems although theoretical understanding is often limited to the generic features of the situation whereas reliable intervention often needs to take the details into account. Let me sketch three pertinent reasons which have to do with the identification of relevant quantities, the generalization of the results, and with ascertaining their reliability in the face of distortions.

The first reason for drawing on theory is the crucial assistance it offers for the identification of the relevant quantities. Theoretical accounts serve to highlight the influential magnitudes and suggest relations among them. In this way they accomplish a figure-ground distinction which 
is often a prerequisite of fruitful empirical investigation. In the case of giant magnetoresistance, the quantum mechanical (or semi-classical) analysis helped to unfold the space of potentially relevant quantities which could subsequently be studied by measuring the precise relationship that obtains between them. Empirical investigation is facilitated considerably by such a theory-based distinction of potentially relevant factors.

This is not distinctive of applied science but rather characterizes scientific research in general. Examples from the history of science provide ample testimony to the fact that relations which appear obvious with hindsight had escaped the notice of experimenters and were only identified after theoreticians had indicated what to look for. For instance, Brownian motion was studied intensively for decades in search for observational regularities without success. In fact, simple such regularities do govern the phenomenon but they were missed since researchers failed to pay attention to the right quantities. Only after Albert Einstein had predicted these relations in 1905 on the basis of theoretical analysis, they were found in observation.

Second, the generalization of theory-shaped models is much easier than the transfer of phenomenological models to new cases encountered. Phenomenological models are shaped conceptually by the demands of the problem-situation at hand. They are not necessarily completely independent of theory, but they contain comparatively few elements that transcend the particulars of the explanatory challenge to be dealt with. As a result, each such phenomenon needs to be approached on its own terms. For instance, the prediction of the tidal flow of a particular harbor is not based on the known causal mechanism underlying the phenomenon but is rather achieved by performing a Fourier analysis of the tidal oscillations observed in the past. The reason is that the influence of a multiplicity of factors relevant for the quantitative details of tidal flow (such as coastline, water depth, currents) can hardly be assessed on first principles so that the phenomenological analysis is more accurate. The drawback is that results gained by this method cannot be transferred to different coastal areas; the latter need to be addressed completely afresh. By contrast, theory-based models whose empirical shortcomings are rectified by parameter fitting and correction factors can be used for a whole class of phenomena. Without employing generic conceptions, each phenomenon had to be attacked without benefitting from the solution to the former.

This feature comes out clearly in the giant-magnetoresistance case. The standard arrangement is a "current-in-plane" geometry in which the electric current flows parallel to the layers of the sandwich-like structure. A different arrangement is the "current-perpendicular-to-plane" geometry, which underlies the most recent development of computer hard disks since it allows a further shrinking in size. This arrangement can be modeled using the same theoretical approaches as applied to the standard case (Wilholt 2006, 83). By contrast, if this configuration had been ap- 
proached on a purely experimental basis it would have to be captured completely on its own. This shows that empirical investigation is facilitated considerably by a theory-based distinction of potentially relevant factors. Theoretical accounts serve to highlight the influential quantities and suggest relations among them. Experiments are appealed to, subsequently, in order to examine the models and to fill the lacunae left by them.

Third and finally, theories are of outstanding importance when the reliability of a device or procedure is to be secured in the face of distorting factors. Ascertaining reliablity typically demands elucidating the underlying causal mechanism. The history of pharmacological research is replete with examples of this sort. Statements about the therapeutic efficacy of a certain medical drug are initially phrased as "contextualized causal relations." Such relations are restricted to typical or normal conditions and leave the pertinent causal processes out of consideration. "Aspirin relieves headache" is a statement of this sort: it usually holds true but possesses exceptions for particular persons or conditions, and it contains nothing as to how the effect is brought about. If the efficiency of the drug is to be improved or pernicious side-effects are to be controlled, the mechanism of action needs to be cleared up. For instance, research on how to circumvent bacterial resistance against antibiotics is targeted on how the drug acts and how the bacterium responds. Likewise, individualized drug treatment, which is among the foci of contemporary medical research, needs to draw on a thorough understanding of the interaction of the agent with the molecular tissue characteristics of the person at hand. In sum, if perturbations intrude, upholding the desired operation of a procedure demands theoretical penetration.

These considerations suggest that overarching theories rightly continue to conceptually shape models in applied research and thereby structure the account of the phenomena while leaving room for empirical adaptation. In fact, theory structure in applied research is not represented by a scattered collection of isolated accounts. Rather, the local models put to use remain linked up with higher-order accounts (Carrier 2004b, 14). Theoretical integration and the understanding thereby generated are essential in applied research although technological devices and inventions need more than theory and heavily draw on empirical adaptation and correction eventually.

\section{Uses of Understanding in Technology Development}

The first question is where all this leaves us with respect to the contrast between the cascade model and the emergentist approach. In light of the preceding considerations, both positions need to be abandoned and give way to an interactive view which preserves aspects of either position. This interactive view stresses that non-theoretical factors like unexplained properties and corrections play an important role in technology development but also emphasizes that the models used for representing the technologically relevant physical processes are conceptually shaped by 
higher-order theories. It militates against the cascade model that it fails to recognize the importance of situation-specific factors. The interactive view accepts the emergentist claim that nature is multifaceted in character and cannot be accounted for without remainder by comprehensive principles. The wealth of the particulars outweigh the impact of the universal. Yet the emergentist approach overshoots the goal by denying theoretical analysis an essential role in the generation of useful knowledge. The best way to deal with multifarious experience is by bringing to bear universal principles and to correct for their shortcomings by empirical adjustments.

The second question is what kind of relationship between technological change and scientific progress this interactive view suggests. To begin with, the demise of the cascade model implies that there is no monocausal chain leading from a scientific discovery up to a technological innovation. Scientific progress is in no way the only source and stimulus for technological change; it may not even represent a predominant influence. First, on the part of the knowledge input, a major part of technology development draws on empirical generalizations and ad-hoc adjustments rather than on systematic theory, let alone on cutting-edge research findings. Second, it has been pointed out frequently that societal demands and market conditions constitute chief driving forces of technology development and heavily affect pathways of technological change. Institutional structures, social exigencies, market demands, traditions, or perceived weaknesses of existing systems are the determinants of the pathways of technology. Scientific discoveries and scientific progress are conspicuous by their absence. This can be made more explicit by a quick glance at some "radical inventions" of the past which profoundly transformed the technological systems dominant at the time: Alexander Bell and the electric telephone, Henry Ford and the assembly line for automobile production, Ferdinand Graf von Zeppelin and the dirigible airship. The crux to these inventions was not supplied by recent progress in understanding; the knowledge that went into them was fairly standard and well established. They were rather due to an engineering type of creativity: new devices were assembled from existing building blocks and widely available components.

These considerations suggest that the technological dynamics proceeds to a considerable extent decoupled from scientific progress. I will add qualifications later but stress right away that this is no way meant to imply that technological development can proceed without a scientific basis. To be sure, inventions and technological innovations do not, as a rule, draw on more recent scientific discoveries. Still, technological novelites do rest on scientific knowledge. Here is an example.

A recent technology invented at the University of South Carolina and developed into a marketable product by a company serves to monitor the composition of substances (like drugs or food) using spectroscopic means. The procedure is based on the separation of the spectrum of the 
expected active ingredient from the background radiation using filters which transmit light only at some selected frequencies, characteristic of the compounds whose concentration is relevant. The advantage is that this quality control can be performed in real time during the production process and can thus be used for instant correction and adjustment (Nelson 1998).

This technology is buttressed by two pillars of established knowledge, namely, spectral analysis in optics and the mathematical procedure of factor analysis. These two received techniques are combined using a particular array of color filters - which is not a spectacularly sophisticated technology either. It is the novel combination of known elements of scientific knowledge that constituted the technological innovation. Moreover, the combination itself was due rather to tinkering than to deduction. Different setups were tried out and the best one retained (Baird 2006).

This example shows that technology development is, in fact, dependent on science but is not always hot on the heels of scientific progress. The science invoked is of some age but maturity doesn't make knowledge less scientific. Although this invention did not rely on recent findings in basic research, it is essentially science-based. The body of scientific knowledge constitutes a huge reservoir of technological options which can be tapped at various locations, not alone at the more recent additions (Rosenberg 1991, 337). Although scientific progress does not anticipate the pathways of technological development, hardly any significant change in an advanced technology lacks a scientific basis.

While the dependence of technological novelties on established knowledge is in accordance with the cascade model, the latter is deficient in a different respect. It fails to take the effect of technology on science into account. First, the development of new instruments for registration and experimentation repeatedly contributed to opening up new intellectual horizons for science. Second, applied research is not infrequently faced with challenges of a more fundamental bearing. The adequate treatment of practical problems may require to address the fundamental challenges as well. In such cases the necessary basic research may be conducted within applied research projects. This feature I call application innovation. It involves the emergence of theoretically significant novelties within the framework of use-oriented research projects.

A large number of such use-oriented projects in the life sciences address questions of fundamental impact. Consider the revolutionary conceptions of "retrovirus" or "prion" which were both conceived in the context of identifying infectious chains. The reduplication of a retrovirus involves the reversal of the familiar directedness of transcription from DNA to RNA, a directedness which was formerly supposed to be universal. Prions were conceived as infectious proteins which assumedly reproduce without assistance of nucleic acids (DNA or RNA). The initial aim of the pertinent studies was to gain useful knowledge about the spread of diseases but they generated 
a deep-reaching transformation of biological concepts. Pursuing practical questions can have a revolutionary impact on the fundamentals.

Application innovation amounts to a partial vindication of the cascade model. It is true that the cascade model says that the most effective way to foster applied science is to support pure science. The sketched examples suggest to the contrary that stimulation may proceed in the opposite direction. However, the cascade model also says that applied science is in its substance based on basic research. The need to take recourse to theoretical principles in meeting practical challenges is made evident by the formulation of such principles in case they are missing. The temporal relations between science and technology are at odds with the cascade model if application innovation occurs. But the logical relations are yet in agreement with the model (Carrier 2004a, 291).

\section{Science Policy and Technological Benefits}

What remains to be clarified is how the interactive view translates into a science policy and a funding strategy. Such policies are usually directed at supporting technological innovations; sponsors typically aim at useful science or theories for use. The impact and success of such policies depend crucially on how science and technology are connected to each other. Bush's idea that progress in basic research is the prime mover of technology dynamics translates into a funding policy that gives priority to broad basic research. However, the underlying idea is mistaken: technology development is rarely based on cutting-edge basic research. In the short run, technological change is largely independent of progress in fundamental science. On the other hand, technological change hinges essentially on scientific knowledge. Although existing technologies and rules of experience constitute another major repository of technological inventions, science constitutes the pivot of technology development. As a result, stopping basic research means drying up the wells and spoiling the seed for future harvest. Accordingly, it remains true that, in the long run, basic research amounts to plowing the field and cultivating the soil so that it bear fruit later. Yet this is a long-term effect whose time-scale is estimated in decades, not years, the usual time-span of science-funding decisions.

Application innovation suggests that the basic research necessary for sustaining applied research may grow out of applied research itself. In such cases applied research produces on its own the knowledge basis required for the solution of applied problems. It generates scientific innovations rather than drawing on them. Basic research may be inspired by considerations of use (Rosenberg 1990, 169; Stokes 1997, 74). As a matter of fact, all the cases I am aware of in which cutting-edge research was transformed quickly into a technological novelty are "use-inspired basic research" (to use Stokes' phrase; Stokes 1997, 93). The spectrum ranges from the nuclear fission 
reactor to the speedy commercial use of the polymerase chain reaction (PCR). ${ }^{1}$ Giant magnetoresistance likewise resulted in marketable technological devices within a short period. Application innovations represent a gain in understanding achieved in pursuing a practical goal. Under such conditions, the new knowledge bears directly on the issues at hand and promotes the rapid transformation of a scientific accomplishment into a technological novelty. This is the qualification indicated earlier (see sec. 5). In cases of application innovation, the practical relevance of basic understanding tends to come out more quickly and the time lag between scientific discovery and its technological implementation is frequently measured in years, not decades.

The interactive view emerging from this discussion amounts to the claim that the body of scientific knowledge and the existing technologies both constitute most important sources of technology development and that, conversely, technology development may stimulate scientific progress. The network of non-scientific factors involved in technology development makes it clear, on the other hand, that the translation of scientific knowledge into technological change is dependent on a number societal presuppositions. Recent studies have revealed that without a suitably arranged economic framework like grant opportunities and funding options, most of the seemingly promising inventions schemes that grow out of research, remain unexplored and wither away. Mere publication is rarely sufficient for instigating corporate research and development activities. In the majority of practical challenges, the influx from science does not set on spontaneously; rather, the faucet needs to be opened-usually by publicly sponsored incentives. ${ }^{2}$

What does this scheme imply for the initial question whether science policy rather support focused practical research projects or broad epistemic research? In contrast to what was suggested by the Bush report, applied research does not detract from the epistemic aspirations and achievements of science. In general, the fear is unfounded that the search for utility drives out the quest for understanding. On the contrary, not infrequently practical problems bring theoretical challenges in their train which need to be taken care of by epistemic research if the the practical endeavor is supposed to get off the ground. The relationship between epistemic and applied research often transcends a peaceful coexistence and rather approaches mutual reinforcement. What is essential, however, for letting this potential of reciprocal stimulation unfold is to leave room or leisure for hooking up the practical goals with the theoretical framework. Research producing light

${ }^{1}$ The institutional motors of such innovations are often start-up companies, not established corporations which rather emphasize incremental inventions (Etzkowitz 2006, 316).

${ }^{2}$ In this vein, Henry Etzkowitz speaks of an "assisted linear model," which means that the cascade from science to technology needs to be set in motion by active government intervention (Etzkowitz 2006, 314-316, 319). 
and fruit at the same time, to use Bacon's apt phrase, requires the willingness and the freedom to address epistemic challenges as they emerge along the road toward some practical research goal.

\section{References}

Adam, Matthias (2005)

"Integrating Research and Development: The Emergence of Rational Drug Design in the Pharmaceutical Industry," Studies in History and Philosophy of Biological and Biomedical Sciences 36, 513-537.

Adam, Matthias, Martin Carrier \& Torsten Wilholt (2006)

"How to Serve the Customer and Still be Truthful: Methodological Characteristics of Applied Research," Science and Public Policy 33, 435-444.

Bacon, Francis (1620)

Neues Organon I, ed. W. Krohn, lat./dt., Hamburg: Meiner, 1990.

Baird, Davis (2006)

"Engineering Reality," Lecture presented at the Conference on "Science in the Context of Application: Transformations of Academic Research," ZiF, Bielefeld University, Oct. 27, 2006.

Bush, Vannevar (1945)

Science The Endless Frontier. A Report to the President, Washington D.C.: United States Government Printing Office, http://www.nsf.gov/od/lpa/nsf50/vbush1945.htm

Carrier, Martin (2004a)

"Knowledge and Control: On the Bearing of Epistemic Values in Applied Science", in: P. Machamer \& G. Wolters (eds.), Science, Values and Objectivity, Pittsburgh: University of Pittsburgh Press; Konstanz: Universitätsverlag, 275-293.

Carrier, Martin (2004b)

"Knowledge Gain and Practical Use: Models in Pure and Applied Research", in: D. Gillies (ed.), Laws and Models in Science, London: King's College Publications, 1-17.

Carrier, Martin (2006)

"The Challenge of Practice: Einstein, Technological Development and Conceptual Innovation," in: C. Lämmerzahl (ed.), Special Relativity: Will it Survive the Next 100 Years?, Heidelberg: Springer,

Carrier, Martin (2007)

„Wissenschaft im Dienst am Kunden: Zum Verhältnis von Verwertungsdruck und Erkenntniserfolg“, in: B. Falkenburg (ed.), Natur - Technik - Kultur. Philosophie im interdisziplinären Dialog, Paderborn: mentis, 15-55.

Cartwright, Nancy (1994)

"Fundamentalism versus the Patchwork of Laws," in: D. Papineau (ed.), The Philosophy of Science, Oxford: Oxford University Press, 1996, 314-326.

Etzkowitz, Henry (2006)

"The New Visible Hand: An Assisted Linear Model of Science and Innovation Policy," Science and Policy 33, 310-320. 
Morgan, Mary S. \& Margaret Morrison (eds.) (1999)

Models as Mediators. Perspectives on Natural and Social Sciences, Cambridge: Cambridge University Press.

Morrison, Margaret (1999)

"Models as Autonomous Agents," in: Morgan \& Morrison 1999, 38-65.

Morrison, Margaret \& Mary S. Morgan (1999)

"Models as Mediating Instruments," in: Morgan \& Morrison 1999, 10-37.

Nelson, Matthew P. et al. (1998)

"Multivariate Optical Computation for Predictive Spectroscopy," Analytical Chemistry 70, 73-82.

Rosenberg, Nathan (1991)

"Critical Issues in Science Policy Research," Science and Public Policy 18, 335-346.

Sauer, Albrecht (2004)

„Im Wandel der Gezeiten“, Spektrum der Wissenschaft 05/2004, 56-59.

Stokes, Donald E. (1997)

Pasteur's Quadrant. Basic Science and Technological Innovation, Washington D.C.: Brookings Institution Press.

Wilholt, Torsten (2006)

"Design Rules: Industrial Research and Epistemic Merit," Philosophy of Science 73, 66-89.

Winsberg, Eric (2003)

"Simulated Experiments: Methodology for a Virtual World," Philosophy of Science 70, 105-125. 